\title{
Effect of Dimethylaminoethano and Compound Amino Acid on Periostin and Dermatopontin Expression in the Skin of D-galactose Induced Aging Model in Rats
}

\author{
Jie Yu Yang ${ }^{1}$, Su Liư
}

${ }^{1}$ Department of Aesthetic and Plastic Surgery, The Affiliated Hospital of Qingdao University, Qingdao 266003, Shandong, China

Abstract: Objective: To study the effect of dimethylaminoethano (DMAE) and compound amino acid inject (AA) by mesotherapy on Periostin and Dermatopontin expression levels of D-galatose induced chemical skin aging rat. Method: by using 80 randomly selected wistar rats divided into normal control group, the aging control group and the experimental groups. At the $18^{\text {th }}$ day after D-gal induction, the experimental group were treated with intra-dermal microinjection of 0.2\% DMAE + AA, 0.1\% DMAE + AA, 0.2\% DMAE, 0.1\% DMAE, AA and NS on bilateral buttocks respectively, once a week for 4 consecutive weeks, after treatments for 42 days, observe the histological changes of dermal thickness, hydroxyproline, and expression of Periostin and Dermatopontin in every individual group. Results: Mesotherapy with intradermal co-injection of $0.1 \%$ DMAE+AA and $0.1 \%$ DMAE + AA have significantly increased the dermal thickness, hydroxyproline content and Periostin expression levels increased significantly, but did not affect the expression of Dermatopontin. Conclusion: by local co-injection of DMAE and AA compound directly under aged rats can effectively reform aged skin tissue structure and the degeneration of collagen, meanwhile it can improve collagen synthesis in aging skin and significantly increases the dermal thickness and collagen content of the skin, and thus prevent skin aging.

Keywords: Dimethylaminoethano; Amino acid; anti-aging; D-galactose; Dermatopontin; Periostin

This study is based on the model of D-galactose induced chemical skin aging rat as research object. According to the Mesotherapy concept, by injecting $0.2 \%$ DMAE+AA, $0.1 \%$ DMAE+AA, $0.2 \%$ DMAE, $0.1 \%$
DMAE, AA and NS on bilateral buttocks of experimental rats under the dermis skin layer, to study the expression of Periostin and Dermatopontin in skin.

I. Materials and Methods 
Effect of Dimethylaminoethano and Compound Amino Acid on Periostin and Dermatopontin Expression in the Skin of D-galactose Induced Aging Model in Rats

\subsection{Experimental Animals}

80 male adult wistar rats weighing $(200 \pm 20) \mathrm{g}$, provided by Institute of Pharmaceutical Sciences (Qingdao, China).

\subsection{Main experimental reagents}

DMAE, D-gal (Shanghai GuoYao Chemical Group Co., Ltd.), 3.48\% AA (Guangdong Litai Pharmaceutical Co., Ltd.), hydroxyproline (Hydroxyproline, Hyp) kit (Nanjing built biotechnology company), PCNA kit (PROTEINTECH, USA), Periostin, Dermatopontin (Beyotime Biotechnology).

Experimental Animals grouping

All experiments are performed in according to the guidelines on Ethical standards for investigation in animals. Table 1 shows the grouping of experimental animals and the grouping of experimental animals. D-gal125mg / $\mathrm{kg}$ was injected into the aging control group and the eexperimental group daily for 42 days. At the $18^{\text {th }}$ day, experimental group were given $10 \%$ chlorinated aldehyde anesthesia, $5 \%$ sodium sulfate for hair removal, in the buttocks on both sides of the production of $3 \mathrm{~cm}$ round tattoo, tattoo skin dermis more uniform injection of $0.1 \%$ DMAE, $0.1 \%$ DMAE + AA, $0.2 \%$ DMAE, $0.2 \%$ DMAE + AA, AA $1 \mathrm{ml}$. Once a week for 4 weeks. All animals are free to eat and drink water. After 42 days, skin specimens are cut and made paraffin sections. The rest part are immediately set to sterile cryopreserved tubes, using liquid nitrogen preservation for later tests.

1.3

Tab 1 Grouping and treatments of the experimental rats $n=10$

\begin{tabular}{llcl}
\hline No. & Experimental Groups & Pretreatment & Intradermal Injection \\
\hline 1 & Aging control group & $125 \mathrm{mg} / \mathrm{kg} \cdot \mathrm{d}$ & \\
2 & Aging NS group & $125 \mathrm{mg} / \mathrm{kg} \cdot \mathrm{d}$ & $\mathrm{NS}$ \\
3 & Aging AA group & $125 \mathrm{mg} / \mathrm{kg} \cdot \mathrm{d}$ & $\mathrm{AA}$ \\
4 & Aging 0.1\%DMAE group & $125 \mathrm{mg} / \mathrm{kg} \cdot \mathrm{d}$ & $0.1 \% \mathrm{DMAE}$ \\
5 & Aging 0.2\%DMAE group & $125 \mathrm{mg} / \mathrm{kg} \cdot \mathrm{d}$ & $0.2 \% \mathrm{DMAE}$ \\
6 & Aging 0.1\%DMAE+AA group & $125 \mathrm{mg} / \mathrm{kg} \cdot \mathrm{d}$ & $0.1 \% \mathrm{DMAE}+\mathrm{AA}$ \\
7 & Aging 0.2\%DMAE+AA group & $125 \mathrm{mg} / \mathrm{kg} \cdot \mathrm{d}$ & $0.2 \% \mathrm{DMAE}+\mathrm{AA}$ \\
8 & Control group & & \\
\hline
\end{tabular}

II. Test method and Test Results

2.1 HE staining: hematoxylin - eosin staining; Using Simple PCI image analysis system, measuring the thickness of dermis, distance between the subcutaneous fat and dermal papilla layer.

2.2 Content of Hydroxyproline (Hyp): the determination of the skin Hyp contents of rats, by the reference of Nanjing Jiancheng bioengineering company kit instructions.

2.3 Using Simple PCI image analysis system, under 40 $\times 10$ magnification, measure the positive staining of fibroblast biopsy from the PNCA immunohistochemistry. At the same magnification, 
Effect of Dimethylaminoethano and Compound Amino Acid on Periostin and Dermatopontin Expression in the Skin of D-galactose Induced Aging Model in Rats

each slice randomly select 5 field views, measure under microscope then take the average value.

2.4 Immunohistochemical PV-9000 two-step method, refer to the test kit instructions. Using the Simple PCI image analysis system, positive staining of immunohistochemistry was measured under $4 \times 10$ magnification. Each slice randomly select 5 view field, measure under microscope then take the average value.

2.5 Statistical analysis: SPSS 22.0 software was used
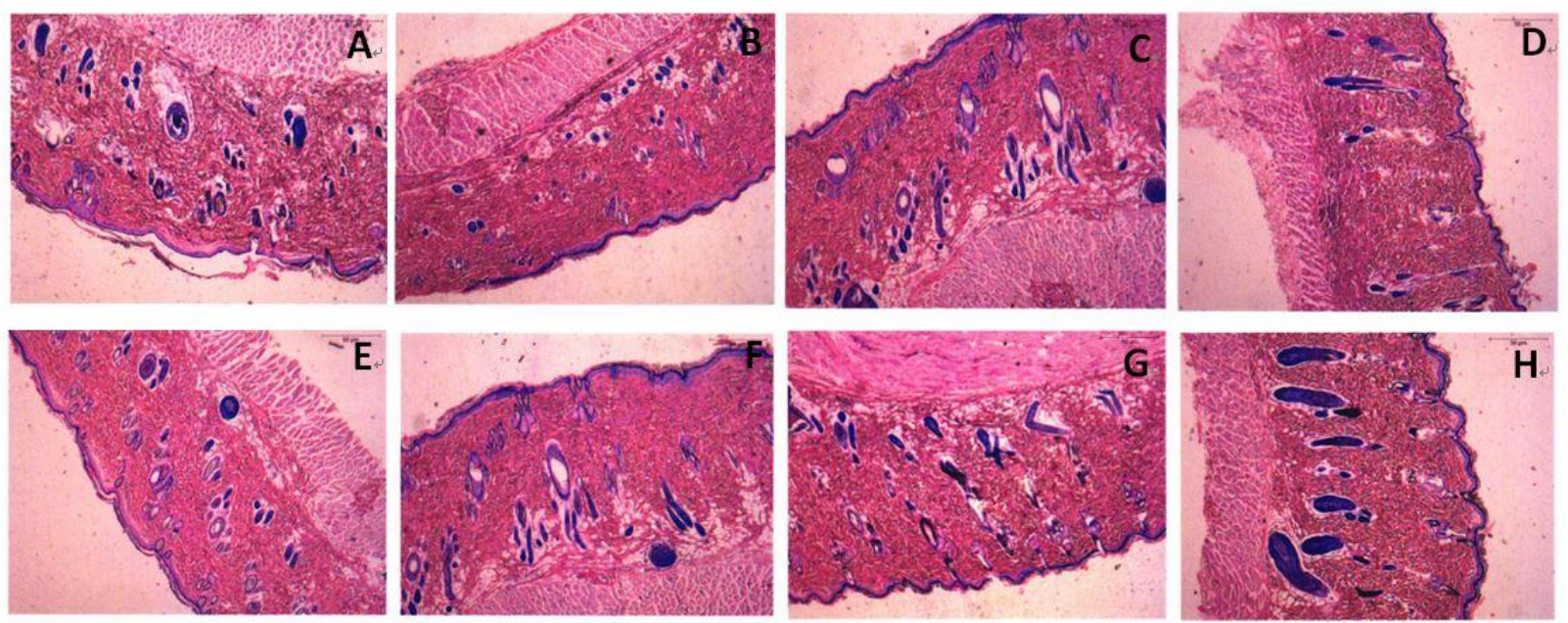

Fig 1. Pictures were taken from each section under $200 \times$ magnification (H\&E). A. Aging control group; B. Aging NS Group; C. Aging AA Group; D. Aging $0.1 \%$ DMAE group; E. Aging 0.2\% DMAE group; F. Aging 0.1\% DMAE + AA Group; G. Aging 0.2\% DMAE + AA; H. Normal Control Group.

to analyze the experimental data. Measurement data using $\mathrm{x} \pm \mathrm{s}$, the difference between the single factor analysis groups, $\mathrm{P}<0.05$ showed that the difference was statistically significant.

\section{Results}

3.1 The animal appearance found that the aging group of rats have reduce body weight, lean body figure, and coat color yellow. Characteristics of aging lead to decreased activities, slow in action, apathetic.
$\mathrm{DMAE}+\mathrm{AA}$ and $0.2 \% \mathrm{DMAE}+\mathrm{AA}$, the above phenomenon is better than the aging control group (Fig.1)

3.3 Dermal thickness: compared with the aging control group, aging group $0.1 \% \mathrm{DMAE}+\mathrm{AA}$ and aging group $0.2 \%$ DMAE + AA has significantly thicker dermis than the aged control group, but were significantly thinner than the normal control group ( $\mathrm{P}<0.01)$, (Table 2). 
Effect of Dimethylaminoethano and Compound Amino Acid on Periostin and Dermatopontin Expression in the Skin of D-galactose Induced Aging Model in Rats

3.4 The effect of DMAE and AA on Hyp content: in normal control group, aging group $0.1 \%$ DMAE +
PCNA in fibroblasts: the expression of PCNA in the normal control group was higher than all aging

Table 2 .

Dermal thickness, Hyp content, expression of Dermatopontin, Periostin in each group.

\begin{tabular}{|c|c|c|c|c|c|}
\hline Groups & ermal Thäckness /um & Content of $\mathrm{Hyp} / \mu \mathrm{m}$ & Periostin $\Delta \Delta C$ t & Dematopontin/ $\triangle \triangle A$ C & PCNApositive cells $\left(/ \mathrm{mm}^{2}\right)$ \\
\hline Aging control group & $392.26=19.56$ & $1.22=0.20$ & $1.00 \pm 0.00$ & $1.00=0.00$ & $26385=6085$ \\
\hline Aging NS group & $403.18=10.95$ & $1.21=0.14$ & $0.0445 \pm 0.121$ & $0.120=0.343$ & $25623 \pm 8792$ \\
\hline Aging AA group & $398.60=10.99$ & $1.27 \pm 0.16$ & $0.0483=0.017$ & $0.110 \pm 0.386$ & $32097 \pm 7434$ \\
\hline Aging $0.1 \% \mathrm{DMAE}$ group & $407.83=20.72$ & $1.27=0.21$ & $0.0380 \pm 0.101$ & $0.119 \pm 0.401$ & $28272 \pm 7109$ \\
\hline Aging $0.2 \% \mathrm{DMAE}$ group & $410.47 \pm 8.37$ & $1.21=0.11$ & $0.0487 \pm 0.158$ & $0.126=0.379$ & $31625 \pm 7902$ \\
\hline Aging $0.1 \% \mathrm{DMAE}+\mathrm{AA}$ group & up $\quad 529.29=26.56$ & $1.50=0.15$ & $0.0426=0.127$ & $0.106=0.057$ & $29831=6953$ \\
\hline Aging $0.2 \% \mathrm{DMAE}+\mathrm{AA}$ group & ap $539.64=18.63$ & $1.55 \pm 0.17$ & $0.0478 \pm 0.083$ & $0.113 \pm 0.745$ & $29697 \pm 6530$ \\
\hline Normal control group & $605.50 \pm 21.02$ & $1.67 \pm 0.21$ & $0.0426=0.015$ & $0.166 \pm 0.860$ & $48646=7370$ \\
\hline
\end{tabular}

AA and aging group $0.2 \% \mathrm{DMAE}+\mathrm{AA}$ has higher content of Hyp than other aging group, have statistical significance $(\mathrm{P}<0.05)$. There was no significant difference between normal control group and aging group $0.1 \% \mathrm{DMAE}+\mathrm{AA}$, aging group $0.2 \% \mathrm{DMAE}+\mathrm{AA}$ and aging group $0.2 \%$ $\mathrm{DMAE}+\mathrm{AA}(\mathrm{P}>0.05)$, Table 2).

3.5 The effect of DMAE and AA on the expression level of Periostin and Dermatopontin: compared with the aging control group, aging 0.1\%DMAE and $0.2 \%$ DMAE + AA group, Periostin expression level is high. Between aging group 0.1\% DMAE and aging group $0.2 \% \mathrm{DMAE}+\mathrm{AA}$, the expression of Periostin has statistical significance $(\mathrm{P}<0.05)$. While between other aging groups, there were no significance difference in the expression level $(\mathrm{P}>0.05)$. There were no significant differences in Dermatopontin expression between normal control group and other aging groups $(\mathrm{P}>0.05)$. Between each aging group, there were no significant differences $(\mathrm{P}>0.05)$, (Table 2).

3.6 The effect of DMAE and AA on expression of group $(\mathrm{P}<0.01)$. There were no statistical significance between each aging groups $(\mathrm{P}>0.05)$.

\section{Discussion}

Skin, as one of the most obvious changing organs in the aging process. While the aging of skin, it is mainly appeared on the surface of skin with shallow wrinkles or deep wrinkles, there are temporary water loss and irreversible water loss on the skin surface and dermis layer. As human ages, skin thickness will progressively decrease. Changes in the dermal structure are the main cause of skin aging.

Mesotherapy was first proposed by French physician Michel Pistor in 1952 [1]. This therapy is achieved by directly injecting a suitable biocompatible and fully absorbable product into the dermis layer to further maintain or restore youthful skin condition while achieving the effect of activating and anti-aging. Mesotherapy is based on the concept of fixed position, fixated layer and fixed quantity, breaking through the skin absorption barrier, which subverts the tradition of 
Effect of Dimethylaminoethano and Compound Amino Acid on Periostin and Dermatopontin Expression in the Skin of D-galactose Induced Aging Model in Rats

beauty in life and variety of beauty care products that are not easily absorb to the epidermis. It is markedly effective, curative effect is obvious, and minimally invasive where recovery period is very short, very suitable for modern busy beauties, in foreign countries are known as "lunch break plastic surgery" therapy. But for its exact curative effect and clear mechanism of action, the literature reports are few, there are still some scholars and experts still have objections on this therapy treatment. [2, 3].

DMAE is an analog of vitamin B choline and a precursor of acetylcholine. Early days was used in treatment of emotional and motor hyperactivity disorders, and now main treating of enhancing memory and improving children's learning and behavioral disorders in the United States. Recent studies find that DMAE can be used as a newer skin anti-aging product, due to a rapid facial improvements and anti-wrinkle curative effects, which has brought to attention. Clinical studies have found that external use of $3 \%$ DMAE gel for 16 weeks has significantly improved prefrontal wrinkles, eye fine lines, lip appearances, and proven that continuous use up to 12 months are well tolerated [4]. External use of 3\% DMAE can obviously increase the skin's firmness $[5,6]$. But study also found that $2.5 \sim 10.0 \mathrm{mmol} / \mathrm{L}$ of DMAE can lead to cultured rabbit fibroblasts, cell will produce large amount of vacuoles; $3 \%$ (337mol/L) DMAE external use also cause rabbits ear skin cell cavity [7]. Gragnani et al [8] found that DMAE inhibited the proliferation of human fibroblasts, causing increased apoptosis, indicate that DMAE has a certain cytotoxicity. At this stage of clinical application, DMAE for external use, such as high doses of the use may cause the corresponding adverse reactions and toxicity $[9,10]$, while also increasing the economic burden. In this study, according to the concept of Mesotherapy, under dermis layer was injected fixed position, fixated layer and fixed quantity, low concentration of DMAE was injected into the treatment site, reducing the dosage and concentration, combined with the simultaneous injection of AA to reduce the intradermal injection of DMAE on the target cell cytotoxicity, where injection site will provide a variety of amino acids as a nutrient.

After the results of this study, we found that the thickness of skin of aging control group was significantly less than the normal rats. On the histological changes, the epidermis was thinned, the layer of cells was reduced, the thickness of the dermis was thinner, collagen fibers were arranged loose, and the density of collagen fiber decreased; slender of collagen bundles and fracture of collagen. In normal rat epidermis can be seen 3 5 layer of cells, arranged dense, corrugated. This study results show that the content of Hyp, collagen and Periostin and Dermatopontin in the skin of the control aging group expression are lower than those of the normal rats. This research, D-gal 125mg/kg was injected subcutaneously in the back of the rat for 42 days, the subcutaneous chemical aging model rat was successfully established.

In this study, we observed the content of Hyp in each group, Hyp is a stable amino acid in collagen, take up $13.4 \%$ in total, by measuring the content, it can clarify the amount of collagen content ${ }^{[11]}$. In this study, it was found that intradermal injection of $0.2 \% \mathrm{DMAE}+\mathrm{AA}$ and $0.1 \%$ DMAE $+\mathrm{AA}$ in aging rats significantly increased the Hyp content in aged rats.

Periostin, has a main function of regulating the 
Effect of Dimethylaminoethano and Compound Amino Acid on Periostin and Dermatopontin Expression in the Skin of D-galactose Induced Aging Model in Rats

formation of fibroblast collagen, proliferating type I collagen. Periostin interacts with type I collagen to regulate the formation of collagen fibrils in connective tissue of dermis. These properties correspond to the phenomena of activated fibroblasts, excessive collagen secretion and so on. It is an extracellular matrix protein, which has the function of promoting cell adhesion and migration. In normal tissues, the expression level is low, mainly during tissue repair, reconstruction process will increase its expression. Dermatopontin (skin bridge protein) has the nature of cell adhesion, as a member of the extracellular matrix, rich in content, meanwhile in vivo regulating cells and extracellular matrix between the information transmission and interaction, enable to maintain the role of internal stability. Previous studies have found that Dermatopontin can promote the formation of collagen fibers, while the new collagen fibers can have a more stable effect. Normally collagen fibers are between $40 \sim 220 \mathrm{~nm}$, with an average of 120 $\mathrm{nm}$. While the presence of Dermatopontin, formed collagen fibers are between $25 \sim 160 \mathrm{~nm}$ in diameter, averaging $80 \mathrm{~nm}$. As a result, Dermatopontin reduced the diameter of the collagen fibers, possibly due to the increase in the total amount of fibers and / or the change in the longitudinal and lateral growth rates of the collagen fibers [12]. Ushio et al were able to observe the effect of Dermatopontin on the formation of collagen fibers in vivo by constructing Dermatopontin knockout mice. Dermatopontin knockout mice reduced the collagen content by $40 \%$. Under the microscope, the diameter and contour of the collagen fibers became irregular. It can be seen that Dermatopontin plays an important role in the accumulation of collagen I the process of maintaining collagen fibers [13]. This study results showed that $0.1 \%$ DMAE + AA and $0.2 \%$ DMAE + AA could significantly increase the expression of Periostin in the dermal layer, and increase the level of Hyp in aging skin, meanwhile dermis layer became thick, it has shown the effect of $0.2 \%$ DMAE + AA compound solution was the most obvious. The expression of Dermatopontin in the aged group was lower than the normal control group. According to the previous in vitro study, Dermatopontin cell adhesion activity has no cell specificity and the affinity between the receptor was low [14]. This suggests that the two groups of 0/1\% $\mathrm{DMAE}+\mathrm{AA}$ and $0.2 \% \mathrm{DMAE}+\mathrm{AA}$ can promote collagen repair in aging skin, can reconstruct the structure of the aging skin, while increasing the collagen content. It can achieve to the level of normal rats; in histological performance the dermis thickness increases, collagen fibers became rich, dense arrangements, corrugated, this has a state of recover to young skin, delay the aging of skin.

The expression of PCNA in the skin was also observed in each experimental groups. PCNA plays an important role in the initiation of cell proliferation and is an important indicator of cell proliferation status [15]. The results showed that there was no significant difference in PCNA between the aging group and the aging control group, which indicated that the injection of the above drugs in the dermis did not induce the proliferation of skin fibroblasts in aged rats.

This experimental study is based on the concept of injection $0.1 \% \mathrm{DMAE}+\mathrm{AA}$ and $0.2 \% \mathrm{DMAE}+\mathrm{AA}$ on the basis of fixed position, fixated layer and fixed quantity, to increase the total amount of collagen and content of Hyp, according to the concept of Mesotherapy. So that the aging skin can restore the original proportion of collagen and structure, which 
Effect of Dimethylaminoethano and Compound Amino Acid on Periostin and Dermatopontin Expression in the Skin of D-galactose Induced Aging Model in Rats

suggests that the two groups of drugs can delay the aging of skin. These two groups of compound solution cannot induce the proliferation of fibroblasts, indicating that the role of anti-aging can only be achieved by promoting the collagen synthesis of fibroblasts. It is safety, no possibility of cell over-proliferate that would lead to mutation or cancer, but its in-depth mechanism of action still remain to have more studies.

\section{References:}

1) Grossman R. The role of dimethylaminoethanol in cosmetic dermatology[J]. Am J Clin Dermatol, 2005,6(1):39-47.

2) Atiyeh BS, Ibrahim AE, Dibo SA. Cosmetic mesotherapy; between scientific evidence, science fiction, and lucrative business [J]. Aesthet Plast Surg. 2008, 32(6):842-849.

3) El-Domyati M, El-Ammawi TS, Moawad O, et al. Efficacy of mesotherapy in facial rejuvenation: a histological and immunohistochmical evaluation [J]. Int J Dermatol, 2012, 51(8):913-919.

4) Grossman RM, Gisoldi EM, Cole C. Long-term safety and effecicacy evaluation of a new skin firming technology: dimethylethanol, $60^{\text {th }}$ Annual Meeting of the Academy of Dermatology [C], 2002:Feb 22-27;New Orleans.

5) Sommerfield B. Randomised, placebo-controlled, double-blind, split-face study on the clinical efficacy of Tricutans on skin firmness [J]. Phytomedicine, 2007, 14(11):711-715.

6) Uhoda I, Faska N, Robert C, et al. Split face study on the cutaneous tensile effect of 2-dimetylaminoethanol (deanol) gel [J]. Skin Res Technol, 2002, 8(3):164-167.

7) Morissette G, Germain L, Marceau F. The antiwrinkle effect of topical concentrated 2-dimethylaminoethanol involves a vacuolar cytopathology $[\mathrm{J}] . \mathrm{Br}$ J Dermatol, 2007, $156(3): 433-439$.

8) Gragnani A, Giannoccaro FB, Sobral CS, et al. Dimethylaminoethanol affects the viability of human cultured fibroblasts [J]. Aesthet Plast Surg, 2007, 31(6):711-718.

9) Grangnani A, Giannoccaro FB, Sobral CS, et al. Dimethylaminoethanol affects the viability of human cultured fibroblasts [J]. Aesthetic Plast Surg, 2007, 31(6):711-718.

10) Grossman R. Gisoldi E. Cole C. Safety and efficacy evaluation of a new skin firming technology: dimethylethanol and tyrosine, 60th Annual Meeting of the Academy of Dermatology; 2002 Feb 22-27: New Orleans.

11) Zhou Hong-bo,Wu Wei,Wang Shi-han,Wu Jing-dong. Effect of Gynostemma on aged skin tissue.[J]. Chinese Journal of Medical Aesthetics and Cosmetology, 2010, 16(6):401-403

12) MacBeth J, Shackleton D, Hulmes D. Tyrosine-rich acidic matrix protein (TRAMP) accerlerates collagen fibril formation. J Biol Chem, 1993, 268(26):19826-19832.

13) Takeda U, Utani A, Adachi E, et al. Targeted disruption of dermatopontin causes abnormal collagen fibrillogenesis, J. Invest Dermatol, 2002, 119(3):678-683.

14) Yang Chen, Guo Mu-Yi, Extracellular New Member-Dermatopontin [J]. Foreign medical Sciences (Section of pathophysiology and Clinical Medicine, 2001, 21(4): $272-273$

15) Wang Qi, Wang Run-he, Hu Qiang, Jin jun-hua. Study of the expression of CD44v6 and PCNA in cutaneous squamous cell carcinoma and cutaneous basal cell Carcinoma. [J]. Chinese Journal of Aesthetic Medicine, 2010, 19(10):1487-1489. 